\title{
OSSOS. V. Diffusion in the Orbit of a High-perihelion Distant Solar System Object
}

\author{
Michele T. Bannister ${ }^{1,2,3}$, Cory Shankman ${ }^{3}$, Kathryn Volk ${ }^{4}$, Ying-Tung Chen (陳英同 $)^{5}$, Nathan Kaib ${ }^{6}$, Brett J. Gladman ${ }^{7}$, \\ Marian Jakubik $^{8}$, J. J. Kavelaars ${ }^{2,3}$, Wesley C. Fraser ${ }^{1}$, Megan E. Schwamb ${ }^{9}$, Jean-Marc Petit ${ }^{10}$, \\ Shiang-Yu Wang ${ }^{5}$, Stephen D. J. Gwyn ${ }^{2}$, Mike Alexandersen ${ }^{5}$, and Rosemary E. Pike \\ ${ }^{1}$ Astrophysics Research Centre, Queen's University Belfast, Belfast BT7 1NN, UK; michele.t.bannister@gmail.com \\ ${ }^{2}$ NRC-Herzberg Astronomy and Astrophysics, National Research Council of Canada, \\ 5071 West Saanich Road, Victoria, British Columbia V9E 2E7, Canada \\ ${ }^{3}$ Department of Physics and Astronomy, University of Victoria, Elliott Building, 3800 Finnerty Road, Victoria, BC V8P 5C2, Canada \\ ${ }_{5}^{4}$ Lunar and Planetary Laboratory, University of Arizona, 1629 E University Boulevard, Tucson, AZ 85721, USA \\ ${ }^{5}$ Institute of Astronomy and Astrophysics, Academia Sinica; 11F of AS/NTU Astronomy-Mathematics Building, \\ Nr. 1 Roosevelt Road, Sec. 4, Taipei 10617, Taiwan, R.O.C. \\ ${ }^{6}$ HL Dodge Department of Physics \& Astronomy, University of Oklahoma, Norman, OK 73019, USA \\ ${ }^{7}$ Department of Physics and Astronomy, University of British Columbia, Vancouver, BC, V6T 1Z1, Canada \\ ${ }^{8}$ Astronomical Institute, Slovak Academy of Science, 05960 Tatranska Lomnica, Slovakia \\ ${ }^{9}$ Gemini Observatory, Northern Operations Center, 670 North A'ohoku Place, Hilo, HI 96720, USA \\ ${ }^{10}$ Institut UTINAM UMR6213, CNRS, Univ. Bourgogne Franche-Comté, OSU Theta F-25000 Besançon, France \\ Received 2017 March 9; revised 2017 April 3; accepted 2017 April 15; published 2017 May 22
}

\begin{abstract}
We report the discovery of the minor planet 2013 SY $_{99}$ on an exceptionally distant, highly eccentric orbit. With a perihelion of $50.0 \mathrm{au}, 2013 \mathrm{SY}_{99}$ 's orbit has a semimajor axis of $730 \pm 40 \mathrm{au}$, the largest known for a highperihelion trans-Neptunian object (TNO), and well beyond those of (90377) Sedna and $2012 \mathrm{VP}_{113}$. Yet, with an aphelion of $1420 \pm 90 \mathrm{au}, 2013 \mathrm{SY}_{99}$ 's orbit is interior to the region influenced by Galactic tides. Such TNOs are not thought to be produced in the current known planetary architecture of the solar system, and they have informed the recent debate on the existence of a distant giant planet. Photometry from the Canada-France-Hawaii Telescope, Gemini North, and Subaru indicate 2013 SY $_{99}$ is $\sim 250 \mathrm{~km}$ in diameter and moderately red in color, similar to other dynamically excited TNOs. Our dynamical simulations show that Neptune's weak influence during 2013 SY $_{99}$ 's perihelia encounters drives diffusion in its semimajor axis of hundreds of astronomical units over 4 Gyr. The overall symmetry of random walks in the semimajor axis allows diffusion to populate $2013 \mathrm{SY}_{99}$ 's orbital parameter space from the 1000 to 2000 au inner fringe of the Oort cloud. Diffusion affects other known TNOs on orbits with perihelia of 45 to 49 au and semimajor axes beyond 250 au. This provides a formation mechanism that implies an extended population, gently cycling into and returning from the inner fringe of the Oort cloud.
\end{abstract}

Key words: Oort Cloud - Kuiper belt objects: individual (2013 SY 99 )

\section{Introduction}

The distant solar system contains a number of small bodies on orbits that are difficult to produce with the currently known planetary architecture. Certain trans-Neptunian objects (TNOs) have orbits with semimajor axes $a \gtrsim 50$ astronomical units (au) that are "detached" from present gravitational interaction with Neptune. These detached objects have perihelia $q \gtrsim 37$ au (Lykawka \& Mukai 2007a; Gladman et al. 2008), much more distant than Neptune's $\sim 30$ au orbit, which prevents substantial gravitational interaction with Neptune even when they are closest to the Sun; they are also not in resonance with Neptune.

The zone of detached orbits, with moderate $a \sim 50-250 \mathrm{au}$, is finely filigreed with high-order mean-motion resonances with Neptune. Simulations show that minor planets could have been emplaced with low efficiency in the era of Neptune's migration (Lykawka \& Mukai 2007b), as far out as a soft boundary in the region $a \lesssim 250 \mathrm{au}$. Methods for this emplacement include resonance sticking in the scattering population or capture into a mean-motion resonance (Brasser \& Morbidelli 2013; Pike et al. 2017), followed by evolution on a periodic orbit of the third kind $^{11}$ (Gomes et al. 2005; Gallardo et al. 2012).

\footnotetext{
11 Often termed "MMR + Kozai", which is not the effect at work here: see discussion in Malhotra et al. (2016).
}

For even larger- $a$ detached orbits, the emplacement history remains more mysterious. The distant semimajor axes of these TNOs are still much smaller than is typical for Oort cloud objects, whose dynamics are significantly affected by Galactic tides. We consider the "inner fringe" of the Oort cloud as $a \sim 1000-2000 \mathrm{au}$; orbits in this quiet zone have placid dynamical interactions, with tides, passing stars, and planetary perturbations only becoming relevant on Gyr timescales. Brasser \& Schwamb (2014) found TNOs with perihelia $q>40 \mathrm{au}$, and semimajor axes $250<a \lesssim 2000 \mathrm{au}$ are currently isolated from gravitational interaction with Neptune or with Galactic tides. Similarly, Kaib \& Quinn (2009) examined the production of long-period comets and found that the production efficiency drops significantly for bodies with $a<3000$ au compared to those with larger semimajor axes. There is no clear consensus on where the Oort cloud dominates; we refer to it as $a \gtrsim 2000$ au (Dones et al. 2004; Kaib \& Quinn 2009).

Minor planets with high-perihelia orbits and $a \lesssim 2000$ au are, thus, only weakly affected by tides and stellar impulses in the current solar environment. Only a few are known: merely six published TNOs have $q>40$ and $a>250$ au (Table 1). We choose to refer to $q>40, a>250$ au minor planets as "extreme" TNOs, avoiding association with particular objects (which may or may not be fully representative of the class) and 
Table 1

Barycentric Orbital Elements of Trans-Neptunian Objects with $q>40$ and $a>250$ au in the International Celestial Reference System at Epoch MJD 57793

\begin{tabular}{|c|c|c|c|c|c|c|c|c|c|c|}
\hline Name & $\begin{array}{c}q \\
(\mathrm{au})\end{array}$ & $\begin{array}{c}a \\
(\mathrm{au})\end{array}$ & $e$ & $\begin{array}{c}i \\
\left({ }^{\circ}\right)\end{array}$ & $\begin{array}{l}\Omega \\
\left({ }^{\circ}\right)\end{array}$ & $\begin{array}{l}\omega \\
\left({ }^{\circ}\right)\end{array}$ & $\begin{array}{l}\varpi \\
\left(^{\circ}\right)\end{array}$ & $\begin{array}{l}\text { Arc } \\
\text { (days) }\end{array}$ & $H_{r}$ & Discovery \\
\hline $2012 \mathrm{VP}_{113}$ & $80.3_{-1.6}^{+1.2}$ & $266_{-17}^{+26}$ & $0.69 \pm 0.03$ & 24.1 & 90.8 & 292.7 & 24.4 & 739 & 4.0 & Trujillo \& Sheppard (2014) \\
\hline $\begin{array}{l}\text { (Sedna) } \\
2003 \mathrm{VB}_{12}\end{array}$ & $76.19 \pm 0.03$ & $507 \pm 10$ & $0.8496 \pm 0.003$ & 11.9 & 144.4 & 311.3 & 96.1 & 9240 & 1.5 & Brown et al. (2004) \\
\hline $2013 \mathrm{SY}_{99}$ & $50.0 \pm 0.1$ & $733 \pm 42$ & $0.932 \pm 0.007$ & 4.2 & 29.5 & 32.2 & 61.7 & 1156 & $6.81 \pm 0.14$ & this work \\
\hline $2010 \mathrm{~GB}_{174}$ & $48.6 \pm 0.1$ & $351 \pm 9$ & $0.862 \pm 0.004$ & 21.6 & 130.7 & 347.2 & 118.5 & 965 & 6.5 & Chen et al. (2013) \\
\hline $2014 \mathrm{SR}_{349}$ & $47.5 \pm 0.2$ & $299 \pm 12$ & $0.841 \pm 0.007$ & 18.0 & 34.9 & 341.2 & 16.3 & 738 & 6.6 & Sheppard \& Trujillo (2016) \\
\hline $\begin{array}{l}(474640) 2004 \\
\mathrm{VN}_{112}\end{array}$ & $47.321 \pm 0.004$ & $316 \pm 1$ & $0.8505 \pm 0.0005$ & 25.6 & 66.0 & 327.1 & 33.1 & 5821 & 6.5 & Becker et al. (2008) \\
\hline $2013 \mathrm{FT}_{28}$ & $43.47 \pm 0.08$ & $295 \pm 7$ & $0.853 \pm 0.004$ & 17.4 & 217.7 & 40.7 & 258.0 & 1089 & 6.7 & Sheppard \& Trujillo (2016) \\
\hline
\end{tabular}

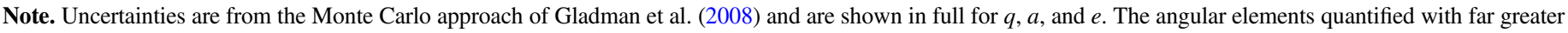
precision are shown only to 0.1 .

with formation mechanisms. The exact boundaries of the class are still being defined.

The extreme dynamical class of TNOs are a signature of a yetto-be-determined aspect of the solar system's architecture or history (Gladman et al. 2002; Brown et al. 2004). Candidate explanations include stellar perturbations on the forming TNO population while the Sun was still in its birth cluster (Brasser et al. 2012), capture of objects from another star in the birth cluster (Kenyon \& Bromley 2004; Jílková et al. 2015), changing proximity to other stars and changing tides as the Sun migrates within the Galaxy (Kaib et al. 2011), gravitational sculpting of distant TNOs during a stellar flyby (Kenyon \& Bromley 2004; Morbidelli \& Levison 2004), and perihelion lifting by an unseen exterior planet, either a "rogue" during its departure from the system (Gladman 2005; Gladman \& Chan 2006) or one still orbiting at present (Gladman et al. 2002; Brown et al. 2004; Gomes et al. 2006; Soares \& Gomes 2013; Trujillo \& Sheppard 2014; Batygin \& Brown 2016).

The extreme-TNO population spend just a small fraction of their orbits near their perihelia, within the magnitude limits attainable by large-aperture telescopes. Analysis of the population is, therefore, particularly sensitive to discovery biases. The proposed formation scenarios cannot yet be observationally distinguished. Schwamb et al. (2010), Brasser et al. (2012), and Trujillo \& Sheppard (2014) find that the stellar cluster origin is consistent with the observed TNOs, but the other scenarios have not been ruled out.

In spite of the low observational probabilities, we recently discovered an exceptional new TNO. In the following sections, we show that $2013 \mathrm{SY}_{99}$ has an orbit with $q=50.0 \pm 0.05 \mathrm{au}$ which has the largest semimajor axis yet found among extreme TNOs. Our four years of observation constrain the semimajor axis to be in the range $694<a<778$ au. Our observations also determine $2013 \mathrm{SY}_{99}$ 's absolute magnitude $\left(H_{r}=6.81 \pm\right.$ 0.14 ) and mildly red color. We model the dynamics of 2013 $\mathrm{SY}_{99}$ in the known solar system and find that $2013 \mathrm{SY}_{99}$ is stable on Gyr timescales. However, its orbit is so weakly bound that very distant interactions with Neptune at perihelion drive a significant random walk in the semimajor axis on timescales of tens of Myr, as in Duncan et al. (1987). We model the evolution of other extreme-TNO orbits and find that gentle evolution via diffusion is a dynamical pathway, linking the populations of the inner fringe of the Oort cloud and the extreme TNOs. We also model 2013 SY99's evolution in a solar system containing a possible distant planet. The model finds that $2013 \mathrm{SY}_{99}$ shows substantial orbital instability or becomes unobservable.

\section{Observations}

$2013 \mathrm{SY}_{99}{ }^{12}$ was found in observations at opposition on 2013 September 29 with the $0.9 \mathrm{deg}^{2}$ field of view MegaPrime imager (Boulade et al. 2003) of the Canada-France-Hawaii Telescope (CFHT) on Maunakea. Three images in the r.3901 filter were taken spanning two hours, targeting the $21 \mathrm{deg}^{2}$ "13BL" survey area of the Outer Solar System Origins Survey (OSSOS). This grid of $3 \times 7$ MegaPrime pointings centered on R.A. $0^{\mathrm{h}} .54^{\prime}$, decl. $+3^{\circ} 50^{\prime}$ was the third of the eight target regions of OSSOS. The observation and data analysis techniques for the survey are detailed in Bannister et al. (2016).

OSSOS completely recovers all TNOs discovered in each target region that are brighter than a limiting magnitude-that region's characterization limit. 89 TNOs were discovered in 13BL, 10 of which were fainter than the characterization limit, including $2013 \mathrm{SY}_{99}$, which had a mean $m_{r}=24.8 \pm 0.3$ at discovery. For 2013 SY $_{99}$ 's rate of apparent sky motion $\left(2\right.$ !" $\left.14 \mathrm{hr}^{-1}\right)$, the characterization limit of the discovery observations was $m_{r}=24.45$. Extrapolating sky motion and magnitude into the less understood uncharacterized region of indeterminate detection efficiency, $2013 \mathrm{SY}_{99}$ was found with a detection efficiency of $\sim 0.09$ (Bannister et al. 2016, Equation (2)). Faint, uncharacterized objects are very difficult to recover and track: they are only imaged if they serendipitously fall within the wide MegaPrime field of view during observations to refine the orbits of the brighter characterized TNOs. The four months of serendipitous observations of 2013 SY $_{99}$ in 2013 implied that it was plausibly on an actively scattering orbit. However, 2013 SY $_{99}$ 's barycentric discovery distance of $60 \mathrm{au}$, substantially greater than the $25<d<52$ au discovery distance of most OSSOS TNOs, flagged it as potentially unusual. Thus, we purposefully recovered 2013 $\mathrm{SY}_{99}$ two years later. The orbital arc for $2013 \mathrm{SY}_{99}$ is presently described by a dense and well-sampled set of 33 observations from 2013 September 5 to 2016 November 4 (Table 3). The astrometry $^{13}$ is measured against the precisely calibrated OSSOS plate solution (Bannister et al. 2016), with median

\footnotetext{
${ }^{12}$ Internal survey designation is uo3191; the TNO has also been referred to as "L91."

13 M.P.E.C. 2017-G55: http://www.minorplanetcenter.net/mpec/K17/ K17G55.html.
} 
Table 2

Published Optical SDSS Broad-band Colors of Trans-Neptunian Objects with $q>40$ and $a>250$ au

\begin{tabular}{|c|c|c|c|}
\hline Name & $g-r$ & $r-i$ & Observations \\
\hline $2012 \mathrm{VP}_{113}$ & $0.70 \pm 0.05$ & $0.32 \pm 0.04$ & $\begin{array}{l}\text { Trujillo \& } \\
\quad \text { Sheppard (2014) }\end{array}$ \\
\hline (Sedna) 2003 VB $_{12}$ & $0.85 \pm 0.03$ & $0.45 \pm 0.03$ & Sheppard (2010) \\
\hline $2013 \mathrm{SY}_{99}$ & $0.64 \pm 0.06$ & $0.46 \pm 0.10$ & this work $\mathrm{k}^{\mathrm{a}}$ \\
\hline (474640) $2004 \mathrm{VN}_{112}$ & $0.69 \pm 0.06$ & $0.24 \pm 0.06$ & Sheppard (2010) \\
\hline
\end{tabular}

Note.

${ }^{\text {a }}$ The two sets of 2013 SY $_{99}$ colors were obtained on different nights (Table 3). The $g-r$ color uses all measurements, weighted by the $\mathrm{S} / \mathrm{N}$ of each measure. Given the extended timespan of the Subaru data, the $r-i$ reported is the $\mathrm{S} / \mathrm{N}$ weighted color of the first two $i$ measures and the first six $r$-band measures, with a 0.05 magnitude uncertainty in quadrature for potential color terms.

residuals of only 0 ". 1 . Unfortunately, $2013 \mathrm{SY}_{99}$ is too faint to be visible in any publicly available archival imaging (Gwyn et al. 2012).

We measured the optical colors of $2013 \mathrm{SY}_{99}$ by imaging with the $8.1 \mathrm{~m}$ Frederick C. Gillett Gemini North Telescope and the $8.2 \mathrm{~m}$ Subaru Telescope, at high airmass (Table 2). A nonconsecutive $r$ and $i$ sequence was observed over four hours with Subaru's Hyper Suprime Cam (HSC; Miyazaki et al. 2012) on 2016 January 10 (Table 3). A consecutive $g$ and $r$ sequence ${ }^{14}$ was observed over two and a half hours on 2016 January 11 with the Gemini Multi-Object Spectrograph (GMOS-N) imager (Hook et al. 2004) with $2 \times 2$ binning (Table 3 ). All observations were made with sidereal tracking, allowing photometric calibration to Sloan Digital Sky Survey (SDSS; Alam et al. 2015) stars. Bias and flat field correction for the GMOS-N data was with the Gemini Observatory Ureka IRAF package and for the HSC data with the package hscPipe. Due to the difficulty of merging the astrometric plate solutions to that of OSSOS from CFHT, the Subaru and Gemini observations do not contribute to our determination of 2013 SY $_{99}$ 's orbit (Section 3.1). Aperture photometry was measured with the moving-object photometry package TRIPPy (Fraser et al. 2016), which includes point-spread function (PSF) fitting and subtraction. We find no evidence for binarity of $2013 \mathrm{SY}_{99}$.

The few measured optical reflectance colors of extreme TNOs range from mildly to considerably redder than solar ${ }^{15}$ $g-r=0.44 \pm 0.02$ (Table 2). We corrected the Gemini instrumental magnitudes to the SDSS by color terms derived in the Colors of OSSOS survey (Schwamb et al., in prep):

$$
\begin{aligned}
g_{G} & =g_{S}-0.146( \pm 0.002)\left(g_{S}-r_{S}\right), \\
r_{G} & =r_{S}-0.048( \pm 0.003)\left(g_{S}-r_{S}\right) .
\end{aligned}
$$

We similarly set the Subaru zero points to the SDSS, adopting an $i$-band color term (R. E. Pike et al. 2017, in preparation); while approximate for HSC, the uncertainty it introduces is significantly smaller than the Poisson uncertainty on the photometry. $2013 \mathrm{SY}_{99}$ 's $r-i=0.46 \pm 0.10$ is slightly red in $r-i$ for a small dynamically excited TNO, but it is still consistent at $1.5 \sigma$ with the $r-i \sim 0.3$ expected from 2013 $\mathrm{SY}_{99}$ 's $g-r=0.64 \pm 0.06$ (Ofek 2012). $2013 \mathrm{SY}_{99}$ 's $g-r$ places it squarely within the $g-r \sim 0.6-0.7$ colors of other

\footnotetext{
14 Fast Turnaround program GN-2015B-FT-26.

15 http://sdss.org/dr12/algorithms/ugrizvegasun/
}

smaller $(H=4-7)$ extreme TNOs, separate from the ultra-red much larger dwarf planet Sedna $(g-r=0.85, H=1.5)$. Colors similarly just redward of solar are seen for comparably sized $H \sim 6-7$ objects in other dynamically excited TNO populations (Sheppard 2010; Fraser \& Brown 2012; Peixinho et al. 2015; Wong \& Brown 2017). The color of 2013 SY $_{99}$ implies that it has a low albedo of $p=0.05 \pm 0.03$ (Fraser et al. 2014; Lacerda et al. 2014).

The observations densely sample the ground-based accessible solar phase angle range $0<\alpha<1^{\circ}$ (Figure 1). This allows for very precise calibration of 2013 SY $_{99}$ 's intrinsic magnitude, to a level normally achieved for asteroids rather than for TNOs. We remeasured 2013 SY $_{99}$ 's photometry with TRIPPy in the CFHT images using the OSSOS-determined centroids and PSF reference stars, with appropriate apertures. The fluxes measured in the Gemini $r$-band were converted into the CFHT $r$-band using the measured color (Gwyn 2008). The phase and variability behavior in the combined CFHT-Gemini photometry was fit in a maximum likelihood sense to the observed magnitudes, adopting a linear phase function and sinusoidal light curve. We adopt the median phase curve slope $\beta$, absolute magnitude $H_{r}$, and light-curve amplitude $\delta$ reported by emcee (Foreman-Mackey et al. 2013) after marginalizing over light-curve period (considering periods 4-24 hr) and phase reference time. $2013 \mathrm{SY}_{99}$ has $\beta=0.38 \pm 0.16 \mathrm{mag} /^{\circ}$, consistent with those of other small TNOs (Rabinowitz et al. 2007). We see variability on few-hour timescales in the Gemini and Subaru data (Table 3), which is entirely consistent with our inferred peak-to-peak amplitude $\delta=0.08$, although we do not attempt to infer a light curve, as the temporal coverage is insufficient. $2013 \mathrm{SY}_{99}$ has $H_{r}=6.81 \pm 0.14$ and is, thus, $\sim 250 \mathrm{~km}$ in diameter.

\section{Orbital Integrations}

\subsection{Present Orbital Properties}

We examined the allowable range of 2013 SY $_{99}$ 's current orbit using the algorithm described in Gladman et al. (2008). This utilizes a Monte Carlo search for the range of orbits in 6D parameter space that are consistent with the available astrometry, accounting for the possible importance of systematics in the astrometric solution. We use a subset of the astrometry, excluding poor-quality images (flagged in Table 3). Because 2013 SY $_{99}$ was discovered near its perihelion, the available four-opposition arc (Section 2) allows us to tightly constrain 2013 SY $_{99}$ 's 50.0 au perihelion distance. However, this four-opposition near-perihelion arc is an extremely small fraction of the TNO's near-twenty-thousandyear orbital period, so its $a / e$ combination is relatively weakly constrained. Very small changes in 2013 SY $_{99}$ 's difficult-toconstrain perihelic speed create large changes in $a$. The Monte Carlo analysis constrains $a$ to be in the range 694-778 au, where the two extremal values in $a$ result in orbit-fit residuals no worse than 1.5 times those of the best-fit orbit. Our uncertainty range is larger than those estimated from the algorithm of Bernstein \& Khushalani (2000), because the search allows for systematic residuals. Other orbital elements are, however, very precisely constrained (Table 1 ).

We integrated the best-fit and the extremal orbits forward in time for $10^{7}$ years with the rmvs 3 subroutine of SWIFT (Levison \& Duncan 1994). On a $10 \mathrm{Myr}$ timescale, 2013 $\mathrm{SY}_{99}$ 's semimajor axis changes by more than $1.5 \mathrm{au}$, which 
would formally classify it as "scattering" per the Gladman et al. (2008) criteria. However, the orbit is metastable on longer solar system timescales.

\subsection{Long-term Evolution in the Known Solar System}

We explored the long-term stability of $2013 \mathrm{SY}_{99}$ in the presence of the four giant planets, both with and without Galactic tides. Figure 2 (top) shows the tide-free evolution of 60 clones of $2013 \mathrm{SY}_{99}$ for $4 \mathrm{Gyr}$ in a 0.5 year time step with the hybrid symplectic/Bulirsch-Stoer algorithm in MERCURY6 (Chambers 1999). As in Shankman et al. (2016), three clusters each of 20 clones were generated, at the $(a, q)$ extremes and at the nominal orbit, per the orbital uncertainties in Table 1. Particles were removed if they reached $a>$ $10,000 \mathrm{au}$; this happened to one out of the 60 total clones.

Duncan et al. (1987) considered the root-mean-square (rms) energy change per perihelion passage for planetary perturbations as a function of $q$. For semimajor axes $a \geqslant 100 \mathrm{au}$, when the orbit is near parabolic, the rms energy change per encounter is $D(x)$. The perihelia stays roughly constant, and the "energy" $x \equiv 1 / a$ undergoes a random walk. The diffusion timescale is $\propto a^{-1 / 2}$, while $D(x) \ll x$. Thus, the energy changes result in comparatively rapid semimajor axis changes that can be modeled as a random walk or diffusion. Following the analysis by Duncan et al. (1987), 2013 SY $_{99}$ receives weak kicks at perihelion with rms dimensionless energy that are of amplitude $10^{-6}-3 \times 10^{-6}$, based on extrapolation of their Figure 1 out to $q=50$ au for low- $i$ orbits. For the $a, e$, and $i$ of $2013 \mathrm{SY}_{99}$, we have directly computed that $D(x) \simeq 1.0 \times 10^{-6}$ via numerical scattering experiments, which agrees very well with the extrapolation. With a perihelion passage every $20 \mathrm{kyr}$, in 4 Gyr the diffusion should drive an energy walk of fractional amplitude $\delta x=\sqrt{200,000} 10^{-6} x$, producing $\delta a=250 \mathrm{au}$, which is in agreement with Figure 2.

The semimajor axis of $2013 \mathrm{SY}_{99}$ is so large that on Gyr timescales, there is semimajor axis diffusion of $100 \mathrm{au}$ or more. Thus, $a$ can change by a factor of two over the age of the solar system. Including Galactic tides in our numerical integrations made no appreciable difference to the simulated evolution, as expected per Kaib \& Quinn (2009). While the fraction of clones spending time with $a>2000$ au is $25 \%$, the planetary energy kicks still dominate over the effects of Galactic tides, because the $\sim 2$ Gyr tidal torquing time for these orbits is longer. The perihelion distance of $2013 \mathrm{SY}_{99}$ is very stable over time; small oscillations in $q$ are secular and proportional to the semimajor axis but are never large enough to strongly couple to Neptune.

The diffusion behavior rules out the possibility of meanmotion resonances (MMR) with Neptune. Any possible Neptune MMR at $>700$ au would have to be higher than 100th order. Such a weak high-order resonance occupies an incredibly thin volume of orbital parameter space: the perturbations of $2013 \mathrm{SY}_{99}$ 's orbit at perihelion would immediately cause a large enough change in $a$ to remove the object from resonance.

$2013 \mathrm{SY}_{99}$ has the lowest inclination of the extreme TNOs found thus far, with $i=4^{\circ} .225 \pm 0^{\circ} .001$; the others have inclinations of $12^{\circ}-26^{\circ}$ (Table 1). $2013 \mathrm{SY}_{99}$ 's inclination remains small: its orbital clones cycled on 100-Myr timescales between $3^{\circ}$ and $6^{\circ}$. The survey fields of OSSOS are predominantly in or near the ecliptic, giving the survey more sensitivity to low- $i$ than to high- $i$ orbits. We used the OSSOS survey simulator (Bannister et al. 2016) to test the observability of a population of test particles by an ensemble of well-characterized TNO surveys: OSSOS's 13AE, 13AO, and 13BL blocks (Bannister et al. 2016), Alexandersen et al. (2016), and CFEPS/HiLat (Petit et al. 2011, 2016). The particles were given orbits from uniform $100<a<$ 1000 au, $45<q<100$ au distributions, with an isotropic inclination distribution from $0^{\circ}$ to $20^{\circ} P(i) \propto \sin (i)$, and random distributions of other angles. We drew $H$ magnitudes with $H_{r} \leqslant$ 8 (recalling $H_{\mathrm{SY} 99}=6.8$ ) from a single slope of $\alpha=0.9$, as is consistent with the observed hot and scattering TNO distributions, prior to the transition to an undetermined form past $H_{r}=8$ (Fraser et al. 2014; Shankman et al. 2016). The simulated detections, shown in Figure 3, are evenly detected at inclinations $3<i<20^{\circ}$, dropping at $i<3^{\circ}$. The $i=4.2$ detection of 2013 $\mathrm{SY}_{99}$ is, thus, reasonable for OSSOS.

\subsection{Diffusion as a Way to Populate the Orbital Phase Space of Extreme TNOs}

The $a$-diffusion exhibited by $2013 \mathrm{SY}_{99}$ due to planetary perturbations works both ways: as well as causing clones of $2013 \mathrm{SY}_{99}$ to migrate outward in $a$, diffusion can be a mechanism to populate 2013 SY $_{99}$ 's orbital parameter space from more distant regions. The diffusion pathway was discussed for $31<q<65 \mathrm{au}, a<500$ au particles by Gallardo et al. (2012) and more briefly for $41<q<43$ au, $a<800$ au particles by Brasser \& Schwamb (2014). Neither tested particles with $q \gtrsim 45$ au in larger semimajor axes ranges.

Our initial population of particles were on inner Oort cloud orbits with random inclinations $0<i<180^{\circ}$ and $1000<a<$ $2500 \mathrm{au} ; 10^{4}$ particles with $45<q<50$ au and a set of smaller batches each of 10 particles with $q$ of $55,58,61,64,67$, and 70 au and $1000<a<2000$, all with random distributions of other elements. We evolved these for $4 \mathrm{Gyr}$ under the influence of the giant planets, including the effects of the Galactic tide and passing field stars. The orbit end-states are shown in Figure 5. After $4 \mathrm{Gyr}$, most of the particles remain with semimajor axes of thousands of au, with a variety of perihelia with $q>30$ au. $13 \%$ have their barycentric distance reach $>200,000$ au and are eliminated from the simulation as ejected. The surviving particles with initial perihelia $q \geqslant 55$ au did not shrink their semimajor axes below their initial $a \geqslant 1000$ au. $25 \%$ of the particles have semimajor axes that diffuse to $a<2000 \mathrm{au}$. At the $4 \mathrm{Gyr}$ point, $1 \%$ of the particles, all from the initial $45<q<50$ au batch, have orbits with $q>40$ au and $a<800$ au. The lowest- $a q>40$ au particle had $a=$ $306 \mathrm{au}$. This shows diffusion from the inner fringe of the Oort cloud can populate the orbital parameter region of $2013 \mathrm{SY}_{99}$.

It is worthwhile to consider if other $45<q<50$ au extreme TNOs like $2010 \mathrm{~GB}_{174}$ and $2014 \mathrm{SR}_{349}$ (Table 1) also show diffusive behavior. Sheppard \& Trujillo (2016) noted semimajor axis mobility for $2013 \mathrm{FT}_{28}$. Particles on orbits in the region showed diffusion in the simulations of Gallardo et al. (2012); they only sampled part of the phase space occupied by the presently known extreme TNOs. We numerically investigate the 4 Gyr orbital evolution of the other extreme TNOs in this $q$ range (including those listed in Table 1) by generating 100 orbital clones of each object from the orbit-fit covariance matrix; we include only the effects of the Sun and the four giant planets in the simulation. Diffusive behavior in the semimajor axis comparable to that of $2013 \mathrm{SY}_{99}$ is frequent among clones of 


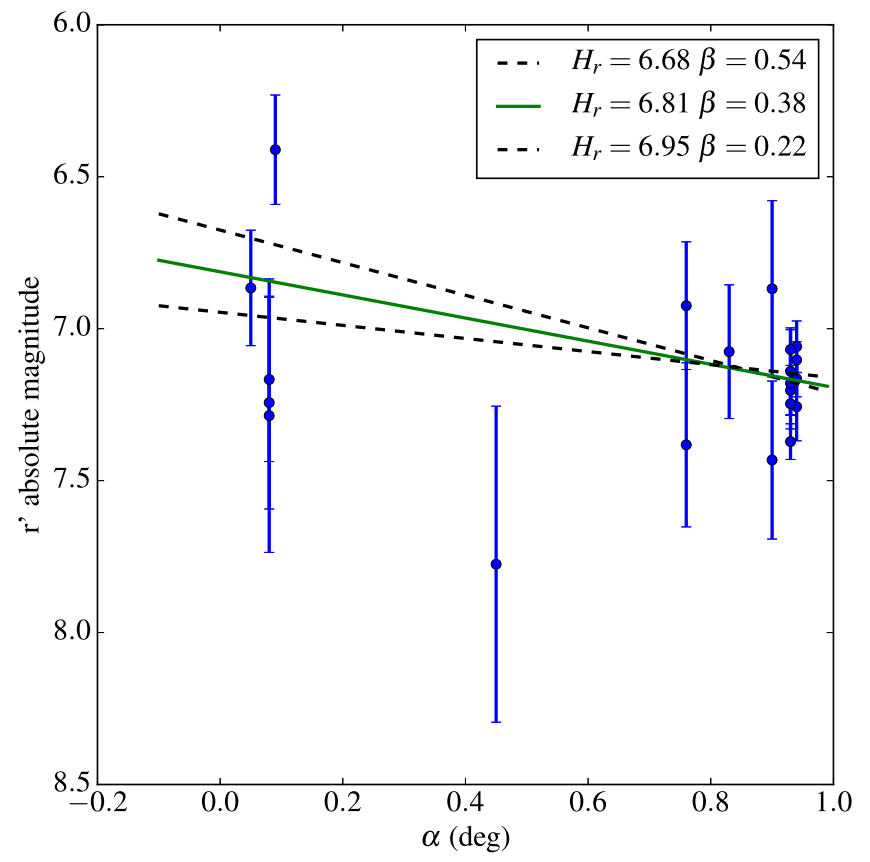

Figure 1. TRIPPy photometry of observations of $2013 \mathrm{SY}_{99}$ in 2013-2016 from CFHT and Gemini, relative to phase angle (Table 3 ). The solid line is the best-fit absolute magnitude $H_{r}$; the dashed lines indicate a $1 \sigma$ uncertainty in the $H_{r}$ fit.

these extreme TNOs, allowing them to evolve to a similar $a, q$ range. An example, (474640) $2004 \mathrm{VN}_{112}$, is shown in Figure 4. These simulations probably underestimate the diffusion and, thus, should not be used for absolute efficiency estimates of the process at this stage. We only simulate planetary perturbations; for completeness, further work should also simulate Galactic tides and passing stars. We also note that the cloning relies on relatively short arcs from the Minor Planet Center. We encourage further Gaia-calibrated (Lindegren et al. 2016) observations of extreme TNOs by the community. All TNOs with clones exhibiting diffusive behavior are marked in Figure 5 (red labels). We note that some clones are capable of diffusion, while other clones are stable on Gyr timescales; the process will be more effective for larger- $a$ TNOs, and the phase spaces of some TNOs (e.g., 2014 $\mathrm{SR}_{349}$ ) show less mobility given their current $a, q$. Sedna and $2012 \mathrm{VP}_{113}$ are not formed by diffusion in the current dynamical environment, which is consistent with previous assessments (Brown et al. 2004; Gallardo et al. 2012; Jílková et al. 2015). However, it is plausible, for the orbit-fit uncertainty parameter space currently occupied by the orbits of other extreme TNOs, that they could be produced by the same inward diffusion from the inner fringe of the Oort cloud that can populate 2013 SY $_{99}$ 's parameter region.

We also find that the clones of some of these lower- $a$ $(a<400 \mathrm{au})$ extreme TNOs show resonance sticking on a variety of timescales from Myr to Gyr. We use the definition for resonance set out in Gladman et al. (2008), i.e., libration of a resonant angle for $10 \mathrm{Myr}$. Diffusive intervals can lead to periods of sticking to a resonance, particularly for $2014 \mathrm{SR}_{349}$. Resonance sticking was not seen for 2013 SY $_{99}$. Objects on the lower $a$ and $q$ sides of the "extreme" region have clones showing stable resonant behavior with Neptune (Figure 5, blue labels). For example, $2000 \mathrm{CR}_{105}(a=224.8 \mathrm{au}, q=44.3 \mathrm{au})$ shows insecure occupation in the 20:1 resonance, as has been long suspected (Gallardo 2006), and some clones of 2013 $\mathrm{GP}_{136}(a=150.2 \mathrm{au}, q=41.0 \mathrm{au})$ librate in a variety of highorder resonances with Neptune. As seen among the phase space occupied by known extreme TNOs in Figure 5, the processes leading to high-order resonance occupation, and of diffusion, show a gradual overlap between their respective regions of dominance.

\subsection{Behavior of $2013 S_{99}$ in a Solar System with a Distant Giant Planet}

It has recently been argued that the apparently non-random orbital orientations of the few known extreme TNOs (defined using varying criteria) is significant. Trujillo \& Sheppard (2014) noted a clustering in the orbits' arguments of pericenter, $\omega$, while Batygin \& Brown (2016) noted a clustering in the longitude of ascending node, $\Omega$, and in the longitude of pericenter, $\varpi$ (the sum of $\omega$ and $\Omega$ ), indicating physical alignment of the orbits. The orientation of 2013 SY $_{99}$ 's orbit in relation to the suggested regions of angular confinement is shown in Figure 2: $2013 \mathrm{SY}_{99}$ is external to the previously noted clustering in $\omega$ and $\Omega$, but within that in $\varpi$. The secular gravitational effects of the giant planets should cause the orbits to precess at different rates, randomizing the orbital angles on $\sim 100$ Myr timescales.

It remains plausible that the complex observational biases in the extreme-TNO discovery surveys have strongly influenced the sample (Sheppard \& Trujillo 2016). Extreme TNOs are affected by the usual biases against the discovery of large- $a$ TNOs: their steep luminosity distribution, the $r^{-4}$ drop in reflected solar flux, and their highly eccentric orbits, placing only a fraction of the orbits near perihelion within the observable depth of even large-aperture telescopes. Layered on this is ephemeris bias: Jones et al. (2010) noted that higheccentricity orbits (c.f. $2013 \mathrm{SY}_{99}$ 's $e=0.93$ ) would be most affected by decisions on tracking observations. At discovery (Section 2), 2013 SY $_{99}$ was too faint to be tracked as part of our magnitude-limited sample, and its orbit implied it was a commonplace scattering TNO. Only its unusual 60 au detection distance raised the possibility it could potentially be on an interesting high-perihelion orbit. If $2013 \mathrm{SY}_{99}$ had instead been at its 50 au perihelion at detection, we would not have worked on tracking it. This illustrates how minor decisions could have biased the known sample. Additionally, surveys' sensitivity to the orientation of orbits varies across the sky. $2013 \mathrm{SY}_{99}$ was detected in an OSSOS block $b=0^{\circ}-3^{\circ}$ below the ecliptic (Section 2). The proximity to the ecliptic induces an observation bias toward detection of objects near perihelion on orbits with argument of perihelion near $0^{\circ}$ or $180^{\circ}$ (Trujillo \& Sheppard 2014; Sheppard \& Trujillo 2016). Unsurprisingly, 2013 SY $_{99}$ 's orbit has $\omega=32^{\circ}$. Lastly, 2013 SY $_{99}$ 's discovery was at opposition near 1. R.A., i.e., in September. This time of year (September-December) experiences typically better seeing (corresponding to deeper limiting magnitudes) in both northern fall and southern spring, continuing a historical seasonal detection bias that can enhance detection at perihelion for surveys, producing clustered longitude of perihelion $\varpi=$ $0^{\circ}-90^{\circ}$. Thus, the simple detection of $2013 \mathrm{SY}_{99}$ does not yet quantitatively assess whether the apparent extreme-TNO clustering arises from a clustered intrinsic population or from observing bias and small-number statistics, and we strongly caution against using it to confirm clustering in the extreme 

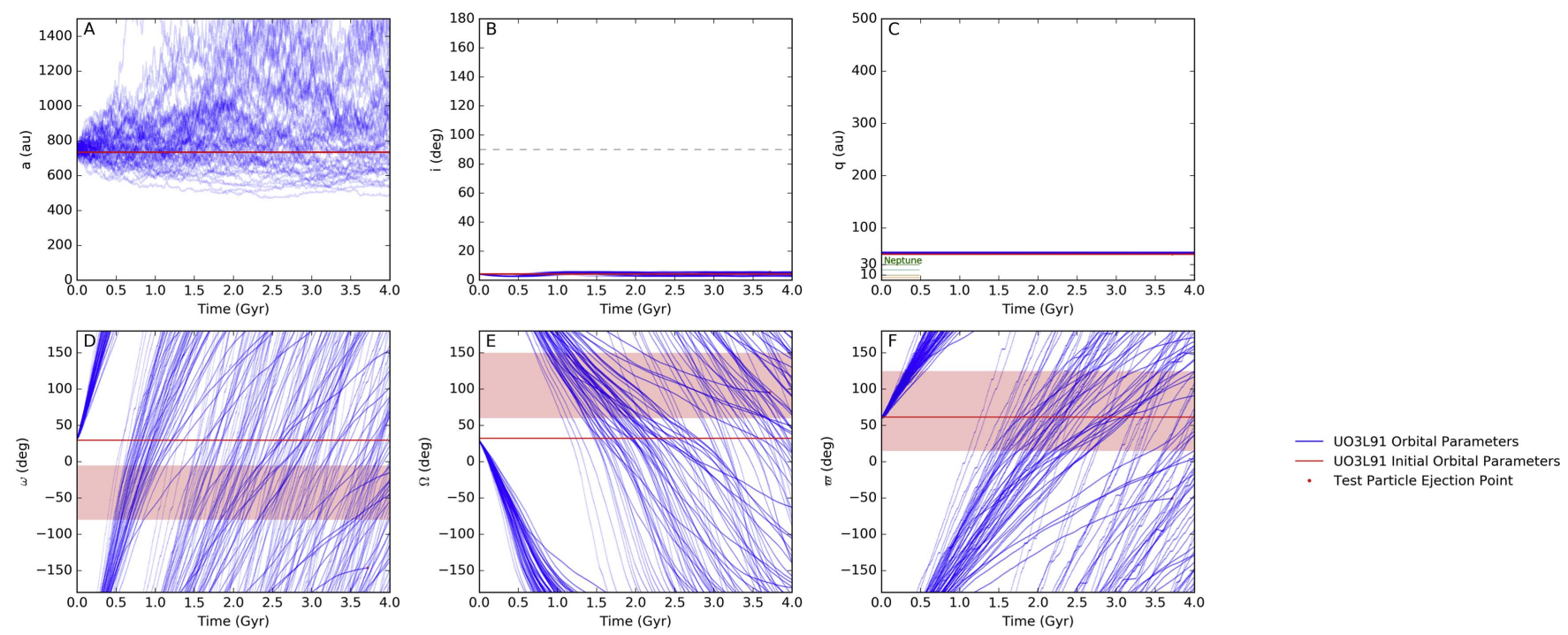

(a)
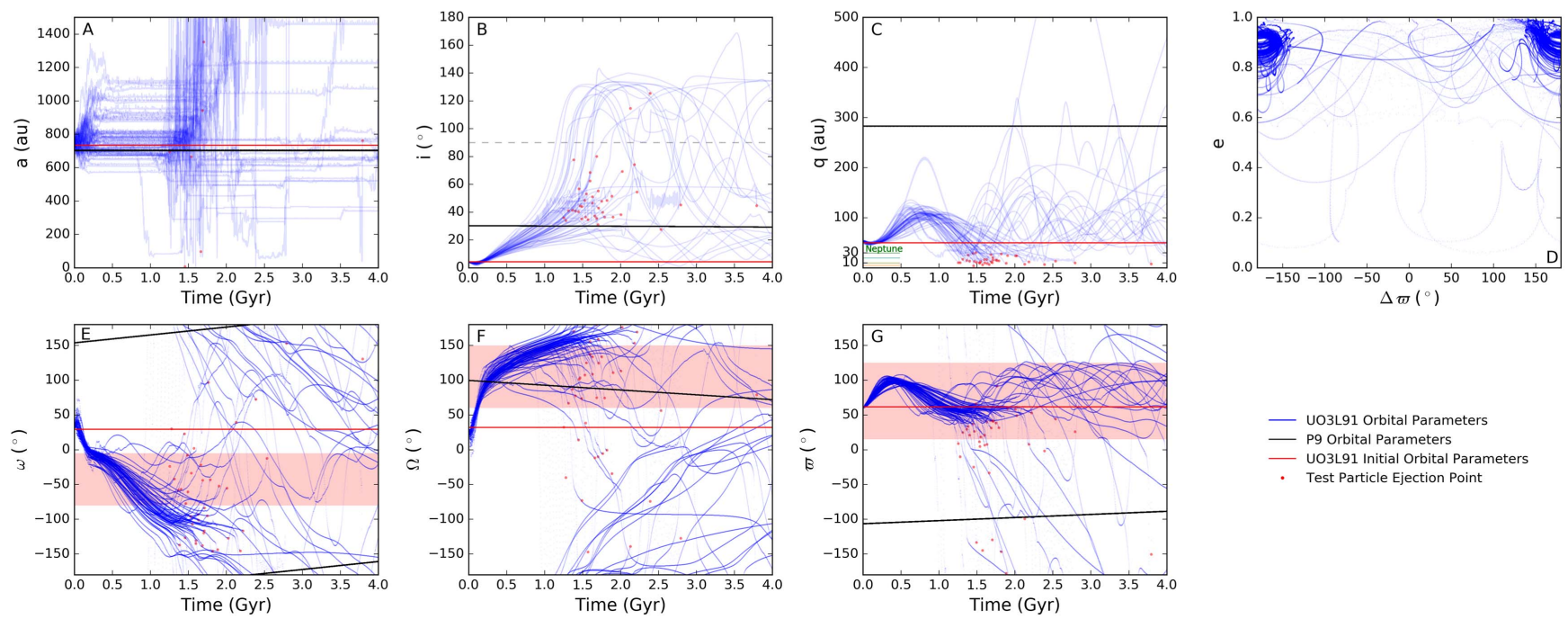

(b)

Figure 2. Orbital evolution over $4 \mathrm{Gyr}$ for 60 clones of $2013 \mathrm{SY}_{99}$ randomly sampled within the uncertainty in its orbital parameters in Table 1 (blue lines). Transparent red circles mark the ejection of a test particle in all panels. The best-fit current orbit of 2013 SY $_{99}$ (red line) and the orbital distances of the four giant planets (lower left corner, Panel C) are indicated in both figures. Pink shading indicates the apparent regions of angular confinement for extreme TNOs, as discussed in Trujillo \& Sheppard (2014) and Batygin \& Brown (2016), for $\omega$ and $\Omega$, 2013 SY $_{99}$ 's current parameters fall outside these regions. (a) Shows evolution in a solar system with four giant planets and no Galactic tides. The perihelia of the clones of $2013 \mathrm{SY}_{99}$ (blue lines) remain constant, while their semimajor axes $\alpha$ diffuse, often out to the inner fringe of the Oort cloud. (b) Displays evolution in a solar system also containing the distant planet of Batygin \& Brown (2016) ( $a=700$ au, $i=30^{\circ}$, $e=0.6, q=280$ au, mass $=10 M_{\oplus}$; black line). A dashed line denotes an inclination of $90^{\circ}$ in panel B. Panel D plots the time evolution of eccentricity vs. the difference of longitude of perihelion, $\varpi$, between the additional planet and the test 2013 SY $_{99}$ clone $\left(\Delta \varpi=\varpi_{\text {Planet }}-\varpi_{\text {clone }}\right)$. The clones of 2013 SY 99 (blue lines) show substantial instability. For the $33 \%$ that survive ejection by Neptune, perihelion is lifted, while inclination steeply increases toward a perpendicular orbit.

TNOs. A forthcoming analysis of the full OSSOS survey will quantitatively examine the survey's observing biases for distant TNOs.

If the clustering is a real feature of the population, it needs to be forced by a dynamical effect. Proposed mechanisms are shepherding from a distant massive planet (Trujillo \& Sheppard 2014; Batygin \& Brown 2016; Brown \& Batygin 2016) or self-gravitation in the initial distant planetesimal disk (Madigan \& McCourt 2016). 2013 SY $_{99}$ is problematic for both models: neither model predicts particles on similar orbits with orbital inclinations as low as that of $2013 \mathrm{SY}_{99}$.

We test the long-term dynamical behavior of $2013 \mathrm{SY}_{99}$ as in Section 3.2, with the addition of the inclined $i=30^{\circ}$ $a=700 \mathrm{au} 10 \quad M_{\oplus}$ planet of Batygin \& Brown (2016) (Figure 2, lower). This simulation does not incorporate tides, as the perturbations due to the planet are orders of magnitude greater (Shankman et al. 2017). The effect of the additional planet is dramatic: on 100-Myr timescales, 2013 SY $_{99}$ 's perihelion distance undergoes significant cyclic variation. For most clones, $q$ drops low enough for the object to experience close encounters with the known giant planets, and is ejected from the solar system. In contrast to the complete stability seen in the known planetary configuration, 40 of the 602013 SY $_{99}$ clones are ejected within a Gyr in a solar system with an additional planet. For the surviving 33\% of the clones of 2013 $\mathrm{SY}_{99}$, their perihelion distances are drawn outward while their 


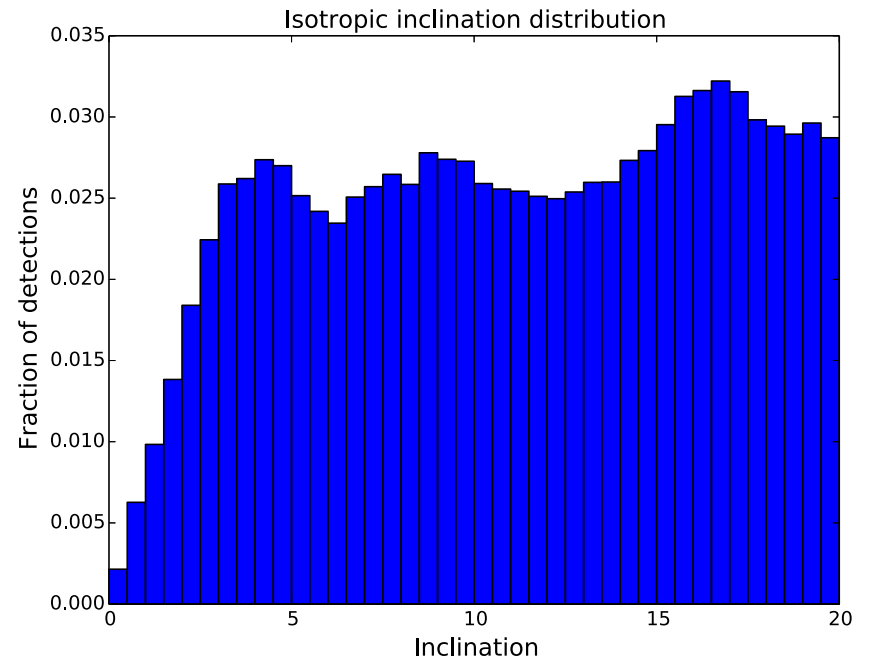

Figure 3. Detectability of a set of particles with uniform $100<a<1000 \mathrm{au}$, $45<q<100$ au distributions and a $P(i) \propto \sin (i) 0^{\circ}-20^{\circ}$ isotropic inclination distribution, to an ensemble of characterized TNO surveys including the first three sky blocks of OSSOS. $2013 \mathrm{SY}_{99}$ has $i=4.2$.

inclinations steeply increase, until the particles are orbiting perpendicular to the plane of the solar system. The inclination and perihelia of the surviving particles cycle widely on nearGyr timescales; these clones also exhibit $\varpi$ shepherding, as in Batygin \& Brown (2016). The $i$ and $q$ evolution of the clones would sharply reduce the detectability of $2013 \mathrm{SY}_{99}$, as the clones spend almost all of their time at high inclinations and larger perihelia. Similar behavior is seen by Shankman et al. (2017) for other extreme TNOs under the Batygin \& Brown (2016) planet parameters. The removal from the detectable volume and high ejection fraction of 2013 SY $_{99}$ clones, thus, requires an enormous population of cycling TNOs to permit the detection of $2013 \mathrm{SY}_{99}$, potentially an $M_{\oplus}$ or more. High-mass estimates are also found in the simulations of Lawler et al. (2016).

With appropriate external planet parameters, $2013 \mathrm{SY}_{99}$ could be phase-protected in a mean-motion resonance (Batygin \& Brown 2016; Malhotra et al. 2016), although this is not strictly required (Beust 2016). However, the present orbit of $2013 \mathrm{SY}_{99}$ is too uncertain to discuss potential resonances with an external planet, as in Malhotra et al. (2016), de la Fuente Marcos \& de la Fuente Marcos (2016), and Millholland \& Laughlin (2017). Several years of further observation will be needed to constrain the semimajor axis to within the width of a potential resonance. There are substantial compounding uncertainties: the technically infinite number of potential resonances, the widths of resonances, the $a$ uncertainties in the extreme-TNO sample, and the flexible range for the potential planet's semimajor axis. Any potential proximity even to low-order resonances is, thus, currently not significant.

\section{Discussion}

We consider how many objects must exist in the orbital parameter space of $2013 \mathrm{SY}_{99}$ for us to have detected it. 2013 $\mathrm{SY}_{99}$ was substantially fainter than the characterization limit at discovery (Section 2). As such, its 9\% detection efficiency is only roughly estimated, which is in contrast to the wellconstrained efficiencies for the vast majority of OSSOS discoveries. Additionally, a derived population estimate must have a factor of ten uncertainty. Proceeding then with caution, the observed existence of any extreme TNOs still implies the presence of a vast unseen reservoir (Gladman et al. 2002; Kaib \& Quinn 2009; Schwamb et al. 2010; Chen et al. 2013).

No model exists for the formation of this population, and the sample size is too small to have a reasonably constrained parametric model. We instead adopt a population deliberately limited in scope, which will be indicative of an underlying population but cannot be robustly tested for rejectability.

We simulated the observability of a 2013 SY $_{99}$-like set of particles to an ensemble of surveys, ${ }^{16}$ as in Section 3.2. The test particle population was a uniformly filled $a-e-i$ box around the uncertainties in the orbit of $2013 \mathrm{SY}_{99}$, assigned random orbit angles and an $H$ magnitude. We used a single slope luminosity function of $\alpha=0.9$ down to $H_{r}=8$, a choice justified in Section 3.2. 2013 SY $_{99}$ 's 0.1 mag variability (Section 2) can only shift the population by a factor of 1.5 . We selected random particles from the model until one was detectable. For us to detect $2013 \mathrm{SY}_{99}$, there need to exist $5 \times 10^{5} H_{r}<8$ objects of $q \sim 50$ and large $a$, to order of magnitude precision. This is consistent with the estimate of

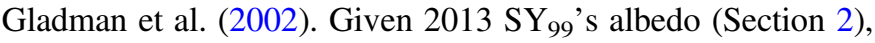
this population with $H_{r}<8$ would have a mass of $0.04 \pm 0.01$ $M_{\oplus}$. Trujillo \& Sheppard (2014) ${ }^{17}$ inferred $0.03 M_{\oplus}$ for a symmetric and larger orbital parameter range $q>50$, $200<a<800$ au population with $\alpha=0.9$.

There are three possibilities for the extreme population:

1. it is in steady state, after primordial emplacement of the inner fringe of the Oort cloud;

2. it was an early emplacement that is decaying over the age of the solar system, like the scattered disk;

3. such objects act like Centaurs, continuously scattered through and rapidly removed from the space between Neptune and a potential outer planet.

We do not assess the last case here. In the steady-state and decaying cases, our simulations in Section 3.3 imply $\sim 1 \%$ of the inner fringe of the Oort cloud has diffused inward and is present in $q>40,300<a<800$ au parameter space. The nature of diffusion means the population is actively, on long timescales, cycling backward and forward. We encourage further investigations of the efficiency of the diffusion process, as it could directly probe the mass resident in the inner fringe of the Oort cloud. For initial emplacement of the population, Dones et al. (2004) found that the giant planets scatter material from the initial protoplanetesimal disk into the whole Oort cloud with an efficiency of $\sim 3 \%$. Kaib et al. (2011) find $\sim 10 \%$ of the Oort cloud mass in the 1000-2000 au region, so the scattering efficiency to there should be a few tenths of a percent. Diffusion as a solo process may, thus, imply an exceptionally massive inner fringe; however, it is unlikely that any one dynamical process solely modifies the population across all solar system history.

We consider the extreme-TNO origin scenarios to see if any formation models for $2013 \mathrm{SY}_{99}$ can be ruled out. A formation scenario must account for both 2013 SY99's detached perihelion and its large semimajor axis.

\footnotetext{
${ }^{16}$ We also made an independent test with the Next Generation Virgo Survey (Chen et al. 2013), which did not provide any stricter constraints.

17 The more extensive continued survey in Sheppard \& Trujillo (2016) does not provide a population estimate for a full range of angles for the orbit ranges discussed here, only an asymmetric clustered-population estimate.
} 

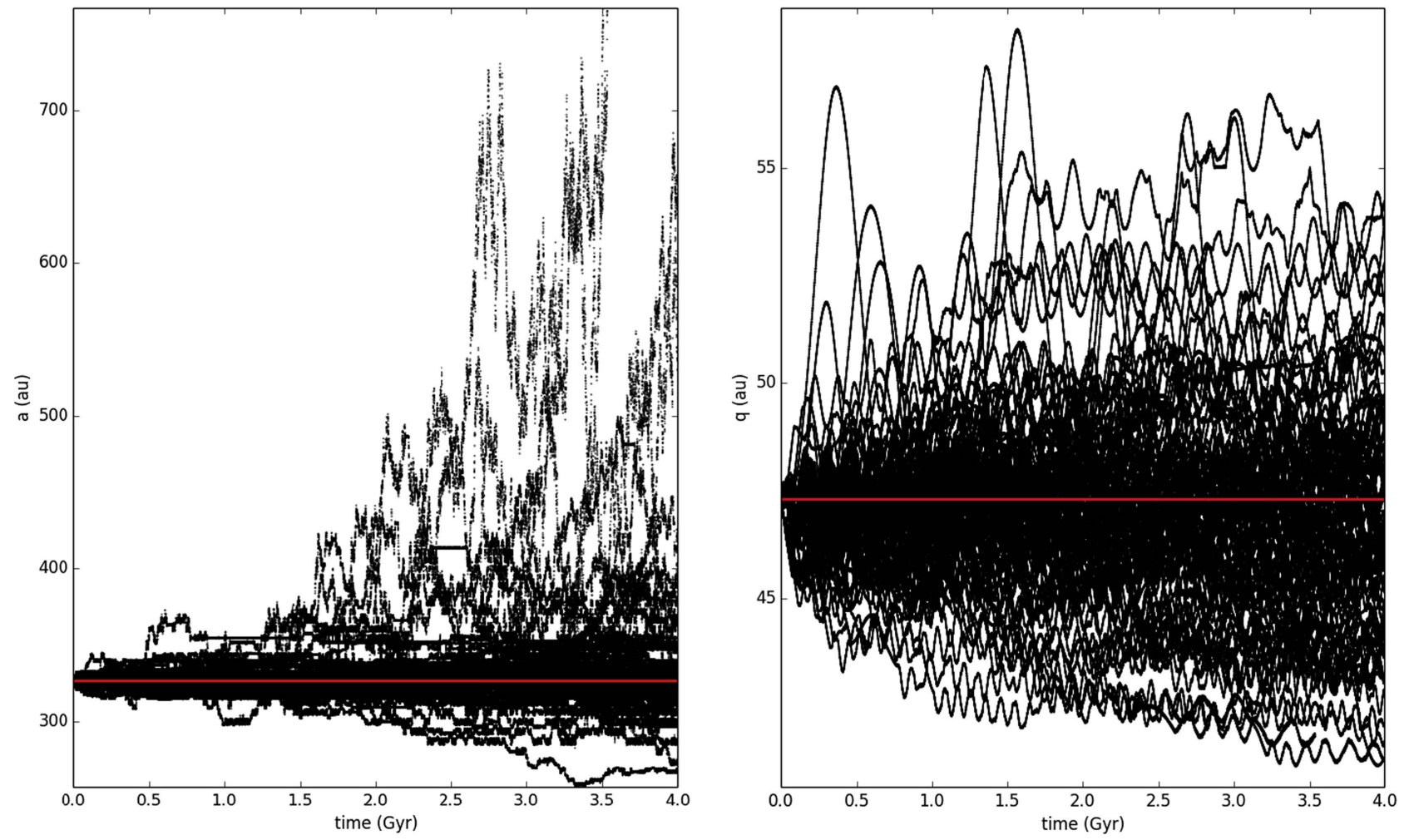

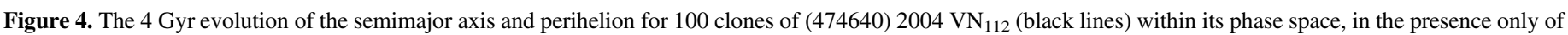
the giant planets. The red line indicates the present best-fit barycentric orbit. This provides an additional example of an extreme-TNO exhibiting diffusion.

The inclination instability of self-gravitating planetesimals (Madigan \& McCourt 2016) cannot yet be excluded; it requires a mass of several $M_{\oplus}$ of objects orbiting with $100<a<$ $10,000 \mathrm{au}$, which is possible, given the population $2013 \mathrm{SY}_{99}$ implies in only $a<2000 \mathrm{au}$.

A presently orbiting distant planet has the advantage of raising perihelia, though we disfavor it due to the loss of $\sim 70 \%$ of 2013 SY $_{99}$ particles and removal of the retained $\sim 30 \%$ of particles from the observationally detectable space, seen in Section 3.4. Scattering from lost embryos/planets remains plausible. Particles covering the orbital parameter space of all extreme TNOs are produced in the rogue planet simulations of Gladman \& Chan (2006), including the decreasing maximum inclination trend with semimajor axis of $i_{\text {imax }}=70^{\circ} \times$ $(100 \mathrm{au} / \mathrm{a})$ present in the observed sample.

Other plausible perihelia-raising mechanisms are perturbation in the Sun's birth cluster, specifically the cluster potential (Jílková et al. 2015), and following the departure of the solar system from the birth cluster, stellar encounters, which can emplace a few tenths of $M_{\oplus}$ (Morbidelli \& Levison 2004). Kaib et al. (2011) found in simulations that only include the standard Galactic tide and passing solar neighborhood stars, minor planets have their orbital perihelia torqued out of the planetary region with $a \sim 1000$ au. Stellar perturbations are a random process, and the most powerful individual stellar encounters the Sun will experience during 4 Gyr provide much more powerful perihelia torquing than the Galactic tide (on the timescale of the encounter or on the Myr timescale of an $a$-diffusing orbit, which is short compared to $4 \mathrm{Gyr}$ ).

We outline a scattering to diffusion scenario for the formation of $2013 \mathrm{SY}_{99}$ that is entirely compatible with the known solar system. An object scatters outward in the initial emplacement of the scattering disk, pushing the semimajor axis of its orbit into the inner fringe of the Oort cloud (e.g., 2014 $\mathrm{FE}_{72}$; Gladman 2005; Levison et al. 2006). At a semimajor axis of a thousand or more au, Galactic tides couple and torque out the orbit's perihelion. Once an object is orbiting with $q=50 \mathrm{au}$ and $a \sim 1000-2000 \mathrm{au}$, it diffuses to a lower- $a$ orbit via planetary energy kicks. A reservoir population of objects must then exist that cycles under diffusion with $q \sim 40-50$ au and $a \sim 1000-2500 \mathrm{au}$. This dynamical pathway is possible in the simplest Oort Cloud model. The shared neutral color of 2013 $\mathrm{SY}_{99}$ and similar-sized objects both in the scattering disk and diffusing extreme TNOs also potentially support a common ancestral origin point in the giant planet region.

We emphasize that this scenario can form the larger- $a$ $q \sim 40-50$ au extreme TNOs, but does not form the $q \sim 80 \mathrm{au}$ TNOs like Sedna: there will be a maximum perihelion above which the kicks from Neptune that permit diffusion to a lower semimajor axis become too weak. It is then worthwhile to consider the perihelia distribution produced by the extremeTNO formation scenarios. Models of stellar perturbation in a cluster environment form Sedna and other $q \sim 80$ au objects as a generic outcome independent of cluster size, with an inner edge to the $q$ distribution (Brasser et al. 2012), which had to be $q \geqslant 75$ au (Schwamb et al. 2010; Sheppard \& Trujillo 2016). Extreme TNOs with perihelia greater than an initial $q_{0}$ are formed by the capture of extra-solar planetesimals during the Sun's cluster birth (Levison et al. 2010; Jílková et al. 2015). In contrast, a distant planet would smoothly lift the perihelia of the extreme TNOs (Gomes et al. 2006; Lawler et al. 2016; Shankman et al. 2017). In our scenario, the initial outward draw of perihelia by tides coupling to large- $a$ scattering objects would also smoothly distribute perihelia.

Figure 5 shows the current absence of extreme TNOs with orbital perihelia in the range 50-75 au (Trujillo \& 


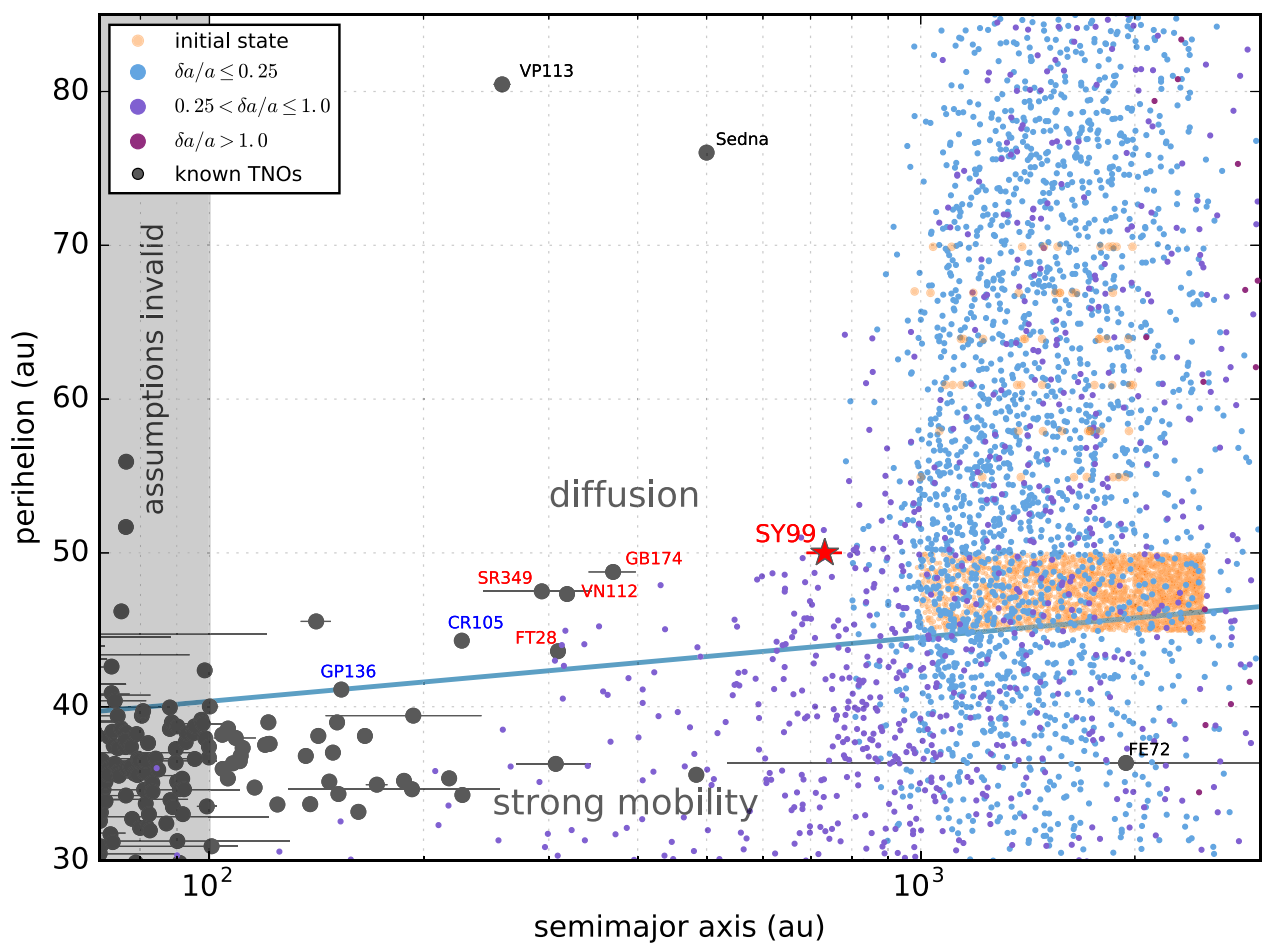

Figure 5. Orbits of $\sim 10^{4}$ particles after $4 \mathrm{Gyr}$ (blue/purple dots), beginning in confined regions of the inner fringe of the Oort cloud (orange dots). Varying degrees of change in $\delta a / a$ after $4 \mathrm{Gyr}$ are indicated by the color of the points (blue to purple). Diffusion, driven by weak Neptune interactions, produces the evolution in $a$ that reaches the parameter space where we find $2013 \mathrm{SY}_{99}$ (red star) and other known extreme TNOs with diffusing orbital clones (red labels). The blue line shows the extrapolated diffusion condition of $\delta a / a=1$ in $4 \mathrm{Gyr}$ for $i=0^{\circ}-30^{\circ}$ (Duncan et al. 1987), a rough boundary between diffusive and strongly mobile actively scattering orbits. At $a<100$ au, the assumptions for diffusive behavior become invalid (shaded region). Some known extreme TNOs have clones in high-order meanmotion resonances with Neptune (blue labels).

Sheppard 2014; Sheppard \& Trujillo 2016). This "perihelion gap" remains, given $2013 \mathrm{SY}_{99}$ has $q=50.0 \mathrm{au}$. A gap in the population excludes the existence of a presently orbiting planet, which would otherwise be cycling minor planets through this region (Shankman et al. 2017). It also rules out other mechanisms for lifting perihelia. OSSOS has found small TNOs at barycentric distances of 50-75 au, and the OSSOS survey simulator indicates that TNOs with perihelia 50-75 au would have been detectable. Testing if their absence is merely a discovery bias is harder; that requires the development of a population model that completely links both this region and another better-understood population, like the scattering disk. However, our scenario for forming 2013 SY $_{99}$ 's orbit does show that for an inner Oort cloud object with $q$ lifted to $\gtrsim 55 \mathrm{au}$, diffusion will be too weak to retract the semimajor axis (Figure 5). Thus, future discoveries with $q \sim 60$ au should have $a \gtrsim 1000$ au.

\section{Conclusion}

Our discovery of an $a=733 \pm 42$ au, $q=50.0$ TNO shows the extreme detached TNO population occupies orbital phase space with much larger semimajor axes than previously seen. We model its orbital evolution over $4 \mathrm{Gyr}$ and find that weak planetary kicks from Neptune at its perihelia still link 2013 SY $_{99}$, and the phase space of other extreme TNOs, to planetary perturbation. Via known processes in the current solar system, TNOs on orbits with $q \sim 45-50$ au and $a \sim 700$ au can be pulled down from semimajor axes beyond 1000 au in the inner fringe of the Oort cloud and move back to similarly large semimajor axes.
We propose a scenario where minor planets scattered outward to the inner fringe of the Oort cloud as part of the emplacement of the scattered disk, Galactic tides lifted the perihelion distances of objects in the inner fringe of the Oort cloud, and diffusion walked their semimajor axes back, forming the population of $2013 \mathrm{SY}_{99}$. This process should form an ongoing cycling of small worlds between the orbital parameter space of $2013 \mathrm{SY}_{99}$ and the $a=1000-2000$ region. The color of $2013 \mathrm{SY}_{99}$ is entirely consistent with a shared primordial population. $2013 \mathrm{SY}_{99}$ is, thus, the closest minor planet to an inner Oort cloud object yet seen. Its existence further supports a substantial $a \sim 1000-2000$ au reservoir of small bodies.

The authors recognize and acknowledge the sacred nature of Maunakea, and appreciate the opportunity to observe from the mountain. This project could not have been a success without the dedicated staff of the Canada-France-Hawaii Telescope (CFHT). CFHT is operated by the National Research Council of Canada, the Institute National des Sciences de l'Universe of the Centre National de la Recherche Scientifique of France, and the University of Hawaii, with OSSOS receiving additional access due to contributions from the Institute of Astronomy and Astrophysics, Academia Sinica, Taiwan. This work is based on observations obtained with MegaPrime/MegaCam, a joint project of CFHT and CEA/DAPNIA; on data produced and hosted at the Canadian Astronomy Data Centre and on the CANFAR VOSpace; on observations obtained at the Gemini Observatory, which is operated by the Association of Universities for Research in Astronomy, Inc., under a cooperative agreement with the NSF on behalf of the Gemini 
Table 3

Observations of 2013 SY $_{99}$ with CFHT, Gemini, and Subaru

\begin{tabular}{|c|c|c|c|c|c|}
\hline $\begin{array}{l}\text { MPC } \\
\text { flag }\end{array}$ & $\begin{array}{c}\text { Time } \\
\text { (Midpoint, UT) }\end{array}$ & Filter & $\begin{array}{l}\text { Exposure } \\
\text { Time (s) }\end{array}$ & $\begin{array}{l}\text { Magnitude } \\
\text { in passband }\end{array}$ & $\begin{array}{c}\text { Solar phase } \\
\alpha\left({ }^{\circ}\right)\end{array}$ \\
\hline \multicolumn{6}{|c|}{ CFHT MegaCam: (photometry: OSSOS, $\uparrow$-flagged: TRIPPy) } \\
\hline & 20130905.42994 & R.3901 & 287 & $25.28 \pm 0.52 \dagger$ & 0.45 \\
\hline V & 20130905.46880 & R.3901 & 287 & $24.74 \pm 0.33$ & 0.45 \\
\hline \multirow[t]{2}{*}{ * } & 20130929.36094 & R.3901 & 287 & $24.76 \pm 0.27 \dagger$ & 0.08 \\
\hline & 20130929.39977 & R.3901 & 287 & $24.98 \pm 0.45 \dagger$ & 0.08 \\
\hline $\mathrm{d}$ & 20130929.44359 & R.3901 & 287 & $24.09 \pm 0.18$ & 0.08 \\
\hline $\mathrm{V}$ & 20130929.44819 & R.3901 & 287 & $24.71 \pm 0.35 \dagger$ & 0.08 \\
\hline $\mathrm{d}$ & 20130929.45297 & R.3901 & 287 & $24.22 \pm 0.23$ & 0.08 \\
\hline $\mathrm{V}$ & 20130929.45785 & R.3901 & 287 & $24.00 \pm 0.18$ & 0.08 \\
\hline $\mathrm{d}$ & 20130929.46414 & R.3901 & 287 & $24.02 \pm 0.22$ & 0.08 \\
\hline $\mathrm{d}$ & 20130929.46913 & R.3901 & 287 & $24.26 \pm 0.29$ & 0.08 \\
\hline $\mathrm{H}$ & 20130929.50184 & R.3901 & 287 & $24.98 \pm 0.39$ & 0.08 \\
\hline V & 20131005.35958 & R.3901 & 287 & $25.41 \pm 0.50$ & 0.03 \\
\hline V & 20131006.36797 & R.3901 & 287 & $24.23 \pm 0.19 \dagger$ & 0.05 \\
\hline V & 20131006.43244 & R.3901 & 287 & $25.25 \pm 0.38$ & 0.05 \\
\hline I & 20131007.35479 & R.3901 & 287 & $23.68 \pm 0.15$ & 0.06 \\
\hline $\mathrm{d}$ & 20131009.49249 & R.3901 & 287 & $23.94 \pm 0.18 \dagger$ & 0.09 \\
\hline \multirow[t]{2}{*}{$\mathrm{d}$} & 20131127.29243 & R.3901 & 287 & $24.84 \pm 0.27 \dagger$ & 0.76 \\
\hline & 20131127.33867 & R.3901 & 287 & $24.43 \pm 0.21 \dagger$ & 0.76 \\
\hline V & $2013 \quad 1205.24094$ & R.3901 & 300 & $24.55 \pm 0.22 \dagger$ & 0.83 \\
\hline \multirow[t]{5}{*}{$\mathrm{V}$} & 20141217.28612 & R.3901 & 500 & $24.28 \pm 0.29 \dagger$ & 0.90 \\
\hline & $201501 \quad 17.25897$ & R.3901 & 387 & $24.94 \pm 0.26 \dagger$ & 0.90 \\
\hline & 20151009.40506 & GRI.MP9605 & 300 & $24.53 \pm 0.13 \dagger$ & 0.90 \\
\hline & 20151009.44841 & GRI.MP9605 & 300 & $24.97 \pm 0.15 \dagger$ & 0.90 \\
\hline & 20151009.49091 & GRI.MP9605 & 300 & $24.59 \pm 0.09 \dagger$ & 0.90 \\
\hline \multirow[t]{6}{*}{$\mathrm{H}$} & 20151117.26167 & GRI.MP9605 & 450 & $\ldots$ & \\
\hline & 20151207.39899 & GRI.MP9605 & 400 & $24.79 \pm 0.15 \dagger$ & 0.84 \\
\hline & 20151231.22796 & GRI.MP9605 & 400 & $24.63 \pm 0.10 \dagger$ & 0.90 \\
\hline & 20160707.58727 & GRI.MP9605 & 300 & $24.81 \pm 0.08$ & 0.98 \\
\hline & 20160708.56666 & GRI.MP9605 & 300 & $24.67 \pm 0.12$ & 0.98 \\
\hline & 20160708.57071 & GRI.MP9605 & 300 & $24.74 \pm 0.13$ & 0.98 \\
\hline \multirow[t]{2}{*}{$\mathrm{d}$} & 20160708.57475 & GRI.MP9605 & 300 & $24.88 \pm 0.14$ & 0.98 \\
\hline & 20161104.31217 & GRI.MP9605 & 450 & $24.66 \pm 0.09$ & 0.46 \\
\hline I & 20161104.37124 & GRI.MP9605 & 450 & $24.46 \pm 0.06$ & 0.46 \\
\hline \multicolumn{6}{|c|}{ Gemini GMOS-N (photometry: TRIPPy) } \\
\hline & 2016-01-07 05:04:57 & r.G0303 & 600 & $24.7069 \pm 0.0572$ & 0.94 \\
\hline & 2016-01-07 05:15:34 & r.G0303 & 600 & $24.6452 \pm 0.0581$ & 0.94 \\
\hline & 2016-01-08 05:11:22 & r.G0303 & 600 & $24.6145 \pm 0.0831$ & 0.94 \\
\hline & 2016-01-08 05:21:58 & r.G0303 & 600 & $24.8176 \pm 0.1110$ & 0.94 \\
\hline & 2016-01-11 04:45:51 & r.G0303 & 600 & $24.6935 \pm 0.1419$ & 0.93 \\
\hline & 2016-01-11 04:56:28 & r.G0303 & 600 & $24.6477 \pm 0.0635$ & 0.93 \\
\hline & 2016-01-11 05:07:06 & r.G0303 & 600 & $24.8037 \pm 0.0566$ & 0.93 \\
\hline & 2016-01-11 05:17:50 & g.G0301 & 600 & $25.2567 \pm 0.0769$ & 0.93 \\
\hline & 2016-01-11 05:28:27 & g.G0301 & 600 & $25.3249 \pm 0.0850$ & 0.93 \\
\hline & 2016-01-11 05:39:04 & g.G0301 & 600 & $25.4567 \pm 0.0998$ & 0.93 \\
\hline & 2016-01-11 05:49:42 & g.G0301 & 600 & $25.4985 \pm 0.1061$ & 0.93 \\
\hline & 2016-01-11 06:00:19 & g.G0301 & 600 & $25.5788 \pm 0.1153$ & 0.93 \\
\hline & 2016-01-11 06:10:57 & g.G0301 & 600 & $25.5309 \pm 0.1145$ & 0.93 \\
\hline & $2016-01-11 \quad 06: 21: 32$ & g.G0301 & 600 & $25.2420 \pm 0.0913$ & 0.93 \\
\hline & 2016-01-11 06:32:09 & g.G0301 & 600 & $25.3515 \pm 0.0957$ & 0.93 \\
\hline & 2016-01-11 06:42:47 & g.G0301 & 600 & $25.6312 \pm 0.1164$ & 0.93 \\
\hline & 2016-01-11 06:53:22 & g.G0301 & 600 & $25.4617 \pm 0.1069$ & 0.93 \\
\hline & 2016-01-11 07:04:05 & r.G0303 & 600 & $24.7828 \pm 0.0810$ & 0.93 \\
\hline & 2016-01-11 07:14:42 & r.G0303 & 600 & $24.8486 \pm 0.0814$ & 0.93 \\
\hline & 2016-01-11 07:25:20 & r.G0303 & 600 & $24.7414 \pm 0.1049$ & 0.93 \\
\hline \multicolumn{6}{|c|}{ Subaru Hyper Suprime Cam (photometry: TRIPPy) } \\
\hline & 2016-01-10 05:13:24 & HSC-I & 300.0 & $24.6001 \pm 0.0939$ & 0.94 \\
\hline & 2016-01-10 05:18:56 & HSC-I & 300.0 & $24.5272 \pm 0.1104$ & 0.94 \\
\hline & 2016-01-10 05:52:21 & HSC-R & 300.0 & $25.0422 \pm 0.0977$ & 0.94 \\
\hline & 2016-01-10 05:57:54 & HSC-R & 300.0 & $25.3657 \pm 0.1394$ & 0.93 \\
\hline
\end{tabular}


Table 3

(Continued)

\begin{tabular}{|c|c|c|c|c|c|}
\hline $\begin{array}{l}\text { MPC } \\
\text { flag }\end{array}$ & $\begin{array}{c}\text { Time } \\
\text { (Midpoint, UT) }\end{array}$ & Filter & $\begin{array}{l}\text { Exposure } \\
\text { Time (s) }\end{array}$ & $\begin{array}{l}\text { Magnitude } \\
\text { in passband }\end{array}$ & $\begin{array}{c}\text { Solar phase } \\
\alpha\left(^{\circ}\right)\end{array}$ \\
\hline & 2016-01-10 06:03:30 & HSC-R & 300.0 & $25.0390 \pm 0.1285$ & 0.93 \\
\hline & 2016-01-10 06:09:02 & HSC-R & 300.0 & $25.0496 \pm 0.1157$ & 0.93 \\
\hline & 2016-01-10 06:14:34 & HSC-R & 300.0 & $24.9846 \pm 0.0952$ & 0.93 \\
\hline & 2016-01-10 06:20:12 & HSC-R & 300.0 & $24.8008 \pm 0.1086$ & 0.93 \\
\hline & 2016-01-10 07:49:23 & HSC-R & 300.0 & $24.6847 \pm 0.1009$ & 0.93 \\
\hline & 2016-01-10 07:54:58 & HSC-R & 300.0 & $24.8982 \pm 0.1622$ & 0.93 \\
\hline & 2016-01-10 08:00:31 & HSC-R & 300.0 & $24.7119 \pm 0.1097$ & 0.93 \\
\hline
\end{tabular}

partnership: the National Science Foundation (United States), the National Research Council (Canada), CONICYT (Chile), Ministerio de Ciencia, Tecnología e Innovación Productiva (Argentina), and Ministério da Ciência, Tecnologia e Inovação (Brazil); and on data collected with the Subaru Telescope, which is operated by the National Astronomical Observatory of Japan. M.T.B. acknowledges support from UK STFC grant ST/L000709/1, the National Research Council of Canada, and the National Science and Engineering Research Council of Canada. M.J. acknowledges the support of the Slovak Grant Agency for Science (grant VEGA No. 2/0031/14).

Facilities: CFHT (MegaPrime), Gemini, Subaru.

Software: Python, astropy, trippy, matplotlib, scipy, numpy, emcee, Ureka, SWIFT, MERCURY6.

\section{Appendix \\ Photometry of $2013 \mathrm{SY}_{99}$}

The Appendix contains Table 3.

\section{References}

Alam, S., Albareti, F. D., Allende Prieto, C., et al. 2015, ApJS, 219, 12 Alexandersen, M., Gladman, B., Kavelaars, J. J., et al. 2016, AJ, 152, 111 Bannister, M. T., Kavelaars, J. J., Petit, J.-M., et al. 2016, AJ, 152, 70 Batygin, K., \& Brown, M. E. 2016, AJ, 151, 22

Becker, A. C., Arraki, K., Kaib, N. A., et al. 2008, ApJL, 682, L53 Bernstein, G., \& Khushalani, B. 2000, AJ, 120, 3323

Beust, H. 2016, A\&A, 590, L2

Boulade, O., Charlot, X., Abbon, P., et al. 2003, Proc. SPIE, 4841, 72

Brasser, R., Duncan, M. J., Levison, H. F., Schwamb, M. E., \& Brown, M. E. 2012, Icar, 217, 1

Brasser, R., \& Morbidelli, A. 2013, Icar, 225, 40

Brasser, R., \& Schwamb, M. E. 2014, MNRAS, 446, 3788

Brown, M. E., \& Batygin, K. 2016, ApJL, 824, L23

Brown, M. E., Trujillo, C., \& Rabinowitz, D. 2004, ApJ, 617, 645

Chambers, J. E. 1999, MNRAS, 304, 793

Chen, Y.-T., Kavelaars, J. J., Gwyn, S., et al. 2013, ApJL, 775, L8

de la Fuente Marcos, C., \& de la Fuente Marcos, R. 2016, MNRAS, 460, L64

Dones, L., Weissman, P. R., Levison, H. F., \& Duncan, M. J. 2004, in Comets

II, Oort Cloud Formation and Dynamics, ed. G. W. Kronk (Tucson, AZ: Univ. Arizona Press), 153

Duncan, M., Quinn, T., \& Tremaine, S. 1987, AJ, 94, 1330

Foreman-Mackey, D., Hogg, D. W., Lang, D., \& Goodman, J. 2013, PASP, 125,306

Fraser, W., Alexandersen, M., Schwamb, M. E., et al. 2016, AJ, 151, 158

Fraser, W. C., \& Brown, M. E. 2012, ApJ, 749, 33

Fraser, W. C., Brown, M. E., Morbidelli, A., Parker, A., \& Batygin, K. 2014, ApJ, 782, 100
Gallardo, T. 2006, Icar, 184, 29

Gallardo, T., Hugo, G., \& Pais, P. 2012, Icar, 220, 392

Gladman, B. 2005, Sci, 307, 71

Gladman, B., \& Chan, C. 2006, ApJL, 643, L135

Gladman, B., Holman, M., Grav, T., et al. 2002, Icar, 157, 269

Gladman, B., Marsden, B. G., \& Vanlaerhoven, C. 2008, in The Solar System Beyond Neptune, ed. M. A. Barucci et al. (Tucson, AZ: Univ. Arizona Press), 43

Gomes, R., Matese, J., \& Lissauer, J. 2006, Icar, 184, 589

Gomes, R. S., Gallardo, T., Fernández, J. A., \& Brunini, A. 2005, CeMDA, 91, 109

Gwyn, S. D. J. 2008, PASP, 120, 212

Gwyn, S. D. J., Hill, N., \& Kavelaars, J. J. 2012, PASP, 124, 579

Hook, I. M., Jørgensen, I., Allington-Smith, J. R., et al. 2004, PASP, 116, 425

Jílková, L., Portegies Zwart, S., Pijloo, T., \& Hammer, M. 2015, MNRAS, 453, 3158

Jones, R. L., Parker, J. W., Bieryla, A., et al. 2010, AJ, 139, 2249

Kaib, N. A., \& Quinn, T. 2009, Sci, 325, 1234

Kaib, N. A., Roškar, R., \& Quinn, T. 2011, Icar, 215, 491

Kenyon, S. J., \& Bromley, B. C. 2004, Natur, 432, 598

Lacerda, P., Fornasier, S., Lellouch, E., et al. 2014, ApJL, 793, L2

Lawler, S. M., Shankman, C., Kaib, N., et al. 2016, AJ, 153, 33

Levison, H. F., \& Duncan, M. J. 1994, Icar, 108, 18

Levison, H. F., Duncan, M. J., Brasser, R., \& Kaufmann, D. E. 2010, Sci, 329, 187

Levison, H. F., Duncan, M. J., Dones, L., \& Gladman, B. J. 2006, Icar, 184,619

Lindegren, L., Lammers, U., Bastian, U., et al. 2016, A\&A, 595, A4

Lykawka, P. S., \& Mukai, T. 2007a, Icar, 186, 331

Lykawka, P. S., \& Mukai, T. 2007b, Icar, 192, 238

Madigan, A.-M., \& McCourt, M. 2016, MNRAS, 457, L89

Malhotra, R., Volk, K., \& Wang, X. 2016, ApJL, 824, L22

Millholland, S., \& Laughlin, G. 2017, AJ, 153, 91

Miyazaki, S., Komiyama, Y., Nakaya, H., et al. 2012, Proc. SPIE, 8446, $84460 \mathrm{Z}$

Morbidelli, A., \& Levison, H. F. 2004, AJ, 128, 2564

Ofek, E. O. 2012, ApJ, 749, 10

Peixinho, N., Delsanti, A., \& Doressoundiram, A. 2015, A\&A, 577, A35

Petit, J.-M., Kavelaars, J. J., Gladman, B. J., et al. 2011, AJ, 142, 131

Petit, J.-M., Kavelaars, J. J., Gladman, B. J., et al. 2016, AJ, submitted (arXiv:1608.02873)

Pike, R. E., Lawler, S., Brasser, R., et al. 2017, AJ, 153, 127

Rabinowitz, D. L., Schaefer, B. E., \& Tourtellotte, S. W. 2007, AJ, 133, 26

Schwamb, M. E., Brown, M. E., Rabinowitz, D. L., \& Ragozzine, D. 2010, ApJ, 720, 1691

Shankman, C., Kavelaars, J. J., Gladman, B. J., et al. 2016, AJ, 151, 31

Shankman, C., Kavelaars, J. J., Lawler, S. M., Gladman, B. J., \& Bannister, M. T. 2017, AJ, 153, 63

Sheppard, S. S. 2010, AJ, 139, 1394

Sheppard, S. S., \& Trujillo, C. 2016, AJ, 152, 221

Soares, J. S., \& Gomes, R. S. 2013 A\&A, 553, A110

Trujillo, C. A., \& Sheppard, S. S. 2014, Natur, 507, 471

Wong, I., \& Brown, M. E. 2017, AJ, 153, 145 